\title{
Localization of Quantitative Trait Loci for Bone Mineral Density on Chromosome 13 in the Mongolian Population
}

\author{
Soo Hyun Seo', Hae Jeng Lim ${ }^{1}$, Sejin Ahn', \\ Joseph Lee ${ }^{3}$ and Jong-II Kim ${ }^{1,2 *}$
}

${ }^{1}$ Genomic Medicine Institute, Medical Research Center, Seoul National University, Seoul 110-799, Korea, '2Department of Biochemistry and Molecular Biology, College of Medicine Seoul National University, Seoul 110-799, Korea, ${ }^{3}$ Taub Institute, Columbia University Medical Center, New York, USA

\begin{abstract}
Although the genetic basis for bone mineral density (BMD) has been studied by many groups so far, genes responsible for this complex trait has not been completely revealed. In order to localize quantitative trait loci (QTLs) for BMD variation in Asian population, the study was designed using a group of Mongolian population, a genetically closed population with a homogeneous lifestyle. BMD was measured at the left and right wrists and ankles using DEXA in 1,082 participants from 142 families. Genotyping of 13 polymorphic microsatellite markers on chromosome 13 (average spacing 8-9 cM) and two-point and multipoint linkage analysis were performed. In two-point linkage analysis, we identified two markers, D13S175 (6.03 cM) and D13S265 (68.73 $\mathrm{CM})$ that had LOD scores greater than 1 for left ankle ( $L O D=2.09, L O D=1.49$, respectively). We also found a marker D13S175 $(6.03 \mathrm{cM})$ with a high LOD for left wrist $(\mathrm{LOD}=1.49)$ and the markers D13S265 $(68.73 \mathrm{cM})$ and D13S217 (17.21 cM) for the right wrist (LOD= 1.82, $\mathrm{LOD}=1.62$, respectively). Among these significant marker regions, only two regions at $17 \mathrm{cM}(13 \mathrm{p} 11)$ and 65 cM (13q21) for the right wrist overlapped with major QTLs reported in following multipoint linkage analysis $(\mathrm{LOD}=1.7549, \mathrm{LOD}=1.4462$, respectively). This study provides the possible evidence of the presence of QTLS affecting right wrist BMD in Mongolian populations on $13 p 11$ and 13q21. Modest evidence was also found for genes affecting left ankle and left wrist BMD on 13p13.
\end{abstract}

Keywords: association analysis, bone density, linkage analysis, mongolian population, QTL

${ }^{*}$ Corresponding author: E-mail jongil@snu.ac.kr

Tel +82-2-740-8241, Fax +82-2-741-5423

Accepted 5 September 2009

\section{Introduction}

Osteoporosis is the primary risk factor for osteoporotic fractures which lead to significantly elevated morbidity and mortality in elderly (Streeten et al., 2006). As populations age in both developed and developing world, the magnitude of this global public health problem will become increasingly significant than before (WHO Scientific group 2003). Previous studies have shown that multiple factors influence the peak bone mass, including genes, hormonal influences, lifestyle and environmental exposures (Jouanny et al., 1995). Specifically, calcium intake, physical activity, and BMl may play important roles in peak bone mass, suggesting that nutritional and genetic factors may interact to influence bone modeling. There are several lines of evidence that support the genetic influence on bone mineral density (BMD). Twin studies have shown that BMD is highly heritable, with genes accounting for $60 \sim 88 \%$ of the total variation in BMD (Barthe et al., 1998; Pocock et al., 1987). Familial aggregation studies have shown that the risk of osteoporotic fracture is elevated in the first degree relatives of probands with osteoporosis (Barthe et al., 1998).

Genetic linkage analysis and candidate genes association studies have implicated several chromosomal loci and multiple candidate genes (Jin and Ralston, 2005). To date, more than 10 studies have performed genomewide linkage scans to localize quantitative trait loci (QTLs) that modulate BMD, and these studies have identified several QTLs. However, only a limited number of loci have been replicated across studies. Strongest support for linkage has been observed for chromosome 1 p36 influencing hip BMD (Devoto et al., 2001). In addition, 1q21, 4q32, 12q24, 13q14 and 16p13 have been implicated for BMD. However, only a few studies have performed a genome wide linkage analysis in Asian populations to localize the region that may harbor susceptibility genes for BMD (Hsu et al., 2007).

In the present study, we examined randomly selected extended families from a region of Mongolia to understand the epidemiologic characteristics of bone health indices (BHI) as surrogates for bone mineral density; to assess the magnitude of heritability of $\mathrm{BHI}$; and to perform variance component linkage analysis for chromosome 13 . 


\section{Methods}

\section{Study population}

The study population was recruited from the residents of Orhontuul soum, Selenge aimag, Mongolia. The study using a Mongolian population was designed for its genetically closed population with a homogeneous lifestyle. We studied a total of 1,082 individuals from 142 Mongolian families. As shown in Table 1, the study included a slightly higher proportion of females compared with males $(56.7 \%$ vs. $43.3 \%$, respectively). Ages for participants ranged from 5 to 82 years; $37.6 \%$ in ages $0 \sim 19$, $13.4 \%$ in $20 \sim 29,19.7 \%$ in $30 \sim 39,12.7 \%$ in $40 \sim 49$ and $16.6 \%$ in 50 and up. Female participants were somewhat older than male participants $(30.6$ vs. 27.6 , respectively).

\section{Genotyping}

Venous blood samples were obtained from all study participants and genomic DNA was extracted from the buffy coat of blood leukocytes. Subjects were genotyped at thirteen microsatellite markers with an average spacing of $8.5 \mathrm{cM}$ using $\mathrm{ABI}$ Prism Linkage Mapping set version 2.5 (Applied Biosystems Inc. CA, USA) (Supplementary Table 1). PCR amplification was done and the software $A B I 300$ DNA sequence and GENESCAN 3.7 was used for analysis. Prior to performing linkage analysis, we checked for deviations from Hardy-Weinberg equilibrium and Mendelian inconsistencies in marker data using the PEDCHECK program (O'Connell and Weeks, 1998).

\section{Bone densitometry}

Bone health indices $(\mathrm{BHI})$ as surrogates for bone mineral density (BMD) was measured using a dual energy X-ray absorptiometer (DEXA, Lunar PIXI densitometer, GE Lunar Corp., USA).

BMD was measured at four sites, left and right wrists, and ankles. Data were reported as $\mathrm{BHI}$ in grams $/ \mathrm{cm}^{2}$.

Table 1. Descriptive statistics of families participating in the Mongolian Genome Project on bone density

\begin{tabular}{lccc}
\hline & Male & Female & Total \\
\hline Number of families & & & 142 \\
No. of individual & 468 & 614 & 1,082 \\
Percent of individual (\%) & 43.3 & 56.7 & 100 \\
Age mean (range) & 27.57 & 30.64 & 29.31 \\
& $(5 \sim 82)$ & $(5 \sim 82)$ & \\
\hline
\end{tabular}

\section{Statistical analyses}

We first examined distribution of bone density index by age and sex. We examined each phenotype to see whether it is normally distributed. Because substantial changes were observed by broad age categories, we stratified subjects into 10 year interval, and computed the mean and standard deviation for BMD at the wrists and ankles. T-scores were calculated using the BMD means and standard deviations using the following equation: T-score $=($ BMDsubject - Peak BMD)/SDpeak for ages 20 years and older. Peak adult bone mass was defined as the mean BMD of the age group with the highest mean BMD value. Based on WHO criteria (WHO Scientific group 2003), subjects with a T-score $\leq-2.5$ were considered to have osteoporosis, and those with a T-score between -1 and -2.5 were considered to have osteopenia. All descriptive statistical analyses were performed using SPSS version 12.0.

We performed heritability and linkage analyses as implemented in SOLAR version 4.0.7 (http://www.sfbr.org/ solar/4.0.7.doc). BMD at the four different sites were used as phenotypes. We first computed heritability for each phenotype, while controlling for age, sex and age by sex. For each trait with the estimated trait standard deviation under 0.5 , a correction factor was applied. For left ankle, left wrist, right ankle and right wrist, a correction factor of $8.4,9,8.7$, and 9.7 was multiplied to $B M D$, respectively. We compared the likelihood for a variance component model where the additive genetic variance for a quantitative trait locus (QTL) $\left(\sigma_{\mathrm{q}}^{2}\right)$ was constrained to zero vs. the likelihood for a model where $\sigma^{2}{ }_{q}$ was estimated. We rejected the null hypothesis if

Supplementary Table 1. Genetic Location for Microsatellite Markers on Chromosome 13

\begin{tabular}{cc}
\hline Marker & $\mathrm{cM}^{*}$ \\
\hline D13S175 & 6.03 \\
D13S217 & 17.21 \\
D13S171 & 25.08 \\
D13S218 & 32.9 \\
D13S263 & 38.32 \\
D13S153 & 45.55 \\
D13S156 & 55.85 \\
D13S170 & 63.9 \\
D13S265 & 68.73 \\
D13S779 & 82.93 \\
D13S173 & 93.52 \\
D13S1265 & 98.82 \\
D13S285 & 110.55 \\
\hline
\end{tabular}

*Marker location is based on the marker map by Marshfield (http://research,marshfield.org). 
the twice the difference in the log likelihood yielded a test statistic that is asymptotically distributed as a 50:50 mixture of a $\chi^{2}$ distribution with one degree of freedom and a point mass at zero (Self and Liang, 1987). We then performed two-point and multipoint linkage analysis. To test for linkage, we compared the likelihood for the null hypothesis of no additive genetic variance due to a QTL $\left(\sigma_{\mathrm{q}}^{2}\right.$ ) against the alternative hypothesis that estimates $\sigma^{2}$ q. Twice the difference in the natural log likelihood of these models yields a test statistic, as described above.

\section{Results}

We studied bone health index in 1,452 individuals in 142 pedigrees. These include 452 nuclear families and 592 founders

Table 2 shows that the mean BMD of left wrist was slightly higher than that for right wrist $(0.44$ vs. 0.43 , respectively), and similarly, the mean BMD for left wrist was somewhat higher than that for left ankle (0.44 vs. 0.41 , respectively). As expected, the mean BMD of males were higher than that of females. The peak value of mean BMD in Mongolian women was calculated to compare with prior studies done in Japan and China.

Table 2. Mean bone mineral density

\begin{tabular}{llll}
\hline & Male & Female & Total \\
\hline BMD-LW mean & 0.479 & 0.444 & 0.459 \\
BMD-LA mean & 0.45 & 0.409 & 0.428 \\
BMD-RW mean & 0.467 & 0.428 & 0.445 \\
BMD-RA mean & 0.458 & 0.424 & 0.439 \\
\hline
\end{tabular}

*BMD, bone mineral density; LW, left wrist; LA, left ankle; $\mathrm{RW}$, right writst; RA: right ankle.
Japan had a peak mean BMD value of $0.359 \mathrm{~g} / \mathrm{cm}^{2}$, China $0.451 \mathrm{~g} / \mathrm{cm}^{2}$ and Mongolia $0.485 \mathrm{~g} / \mathrm{cm}^{2}$ which shows Mongolian women to have the highest value.

The polynomial regression equations describe the relation between age and BMD at each site (Supplementary Table 2). The cubic regression model was the simplest model that significantly improved the fit to the data at every site. The best fitting effect $\left(R^{2}\right)$ of the regression model that includes age, age $^{2}$ and age $^{3}$ explains $70.5 \%$ of the observed variation for left wrist, $41.2 \%$ for left ankle, $70.5 \%$ for right wrist, and $41.9 \%$ for right ankle.

Using the definition of osteoporosis by WHO criteria, the prevalence of osteoporosis was approximately threeto four-fold higher in females than in males in this population. For example, $42 \%$ of the female subjects met the criteria of osteoporosis in the ankle compared with $11 \sim 12 \%$ in males (Supplementary Table 3 ).

Variance component linkage analysis for each trait was performed using 13 microsatellite markers. After adjusting for age, sex, and age by sex in the polygenic

Supplementary Table 3. Percentage of osteoporosis at various sites

\begin{tabular}{|c|c|c|c|c|}
\hline Male & Normal & Osteopenia & Osteoporosis & Total \\
\hline Left ankle & $25(55.6 \%)$ & $15(33.3 \%)$ & $5(11.1 \%)$ & 45 \\
\hline Left wrist & 32 (71.1\%) & $11(24.4 \%)$ & $2(4.4 \%)$ & 45 \\
\hline Right ankle & $28(48.3 \%)$ & $23(39.7 \%)$ & $7(12.1 \%)$ & 58 \\
\hline Right wrist & $39(66.1 \%)$ & $15(25.4 \%)$ & $5(8.5 \%)$ & 59 \\
\hline Female & Normal & Osteopenia & Osteoporosis & Total \\
\hline Left ankle & $16(20.3 \%)$ & $30(38.0 \%)$ & $33(41.8 \%)$ & 79 \\
\hline Left wrist & $28(35.9 \%)$ & $35(44.9 \%)$ & 15 (19.2\%) & 78 \\
\hline Right ankle & 15 (15.8\%) & $40(42.1 \%)$ & $40(42.1 \%)$ & 95 \\
\hline Right wrist & $31(32.0 \%)$ & $45(46.4 \%)$ & $21(21.6 \%)$ & 97 \\
\hline
\end{tabular}

Supplementary Table 2. Polynomial regression equations and coefficients of determination for T-score at wrists and ankles

Male

\begin{tabular}{|c|c|c|c|}
\hline Site & Polynomial regression equation & R-square & $\mathrm{p}$-value \\
\hline Left wrist & T-score $=-6.626+0.404($ age $)-0.007(\text { age })^{2}+0.0000354(\text { age })^{3}$ & 0.705 & $<0.001$ \\
\hline Left ankle & T-score $=-4.374+0.338($ age $)-0.008(\text { age })^{2}+0.0000517(\text { age })^{3}$ & 0.412 & $<0.001$ \\
\hline Right wrist & T-score $=-7.365+0.470($ age $)-0.009(\text { age })^{2}+0.0000456(\text { age })^{3}$ & 0.705 & $<0.001$ \\
\hline Right ankle & T-score $=-4.211+0.321($ age $)-0.008(\text { age })^{2}+0.0000501(\text { age })^{3}$ & 0.419 & $<0.001$ \\
\hline \multicolumn{4}{|l|}{ Female } \\
\hline Site & Polynomial regression equation & R-square & $\mathrm{p}$-value \\
\hline Left wrist & T-score $=-6.792+0.486($ age $)-0.010(\text { age })^{2}+0.0000584(\text { age })^{3}$ & 0.676 & $<0.001$ \\
\hline Left ankle & T-score $=-3.987+0.349($ age $)-0.008(\text { age })^{2}+0.0000548(\text { age })^{3}$ & 0.294 & $<0.001$ \\
\hline Right wrist & T-score $=-6.923+0.503($ age $)-0.011(\text { age })^{2}+0.0000601(\text { age })^{3}$ & 0.678 & $<0.001$ \\
\hline Right ankle & T-score $=-4.023+0.352($ age $)-0.009(\text { age })^{2}+0.0000577(\text { age })^{3}$ & 0.329 & $<0.001$ \\
\hline
\end{tabular}




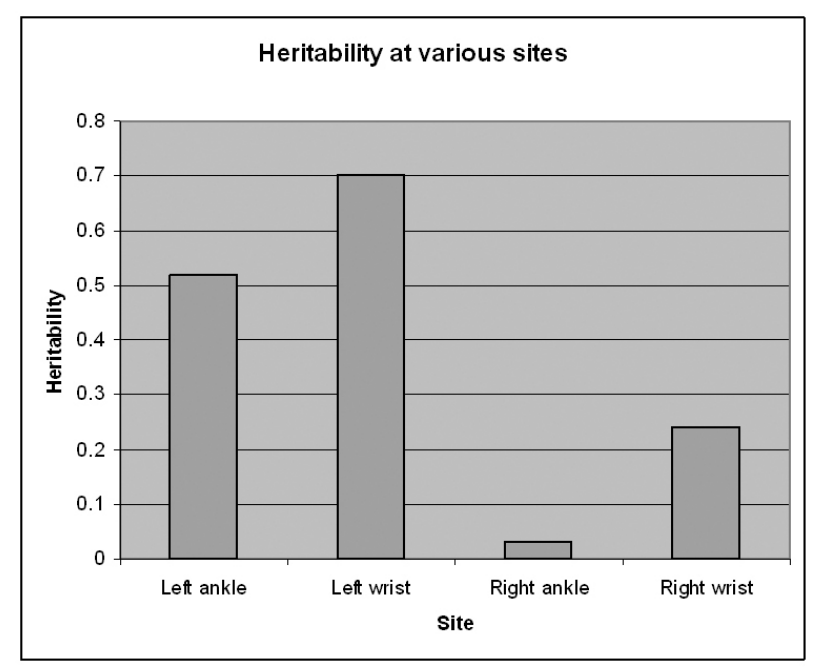

Fig. 1. Heritability of $B M D$ at various sites. Total additive genetic heritability $\left(\mathrm{H}_{\mathrm{r}}^{2}\right)$ for BMD were estimated at four different sites as indicated, using variance component analysis. model, estimates for total additive genetic heritability $\left(\mathrm{H}^{2} \mathrm{r}\right)$ were high for left ankle and left wrist among four different sites. $\mathrm{H}^{2}$ for left ankle was $0.52(\mathrm{p}<0.001)$, left wrist $0.70(p<0.001)$. On the other hand, compared with heritability estimates for left wrist and ankle, $\mathrm{H}_{r}^{2}$ was lower for right ankle $0.03(p=0.25)$ and right wrist 0.24 $(p<0.001)$ (Fig. 1).

Two-point linkage analysis based on thirteen microsatellite markers on chromosome 13 revealed that D13S175, located at $6.03 \mathrm{cM}$, had the highest two-point LOD score of 2.09 for left ankle, 1.49 for left wrist, and 0.42 for right ankle. In addition, D13S265, located at $68.73 \mathrm{cM}$ showed evidence of linkage for the BMD value for right wrist $(L O D=1.82)$, and for left ankle, wrist also (LOD=1.65, $L O D=1.16$, respectively) (Fig. 2).

For the two markers with the high LOD scores, we then examined family-specific LOD scores to see whether these linkage signals were derived from a subset of families. Each analysis showed subsets of families with high LOD scores. In the analysis on left ankle and wrist,
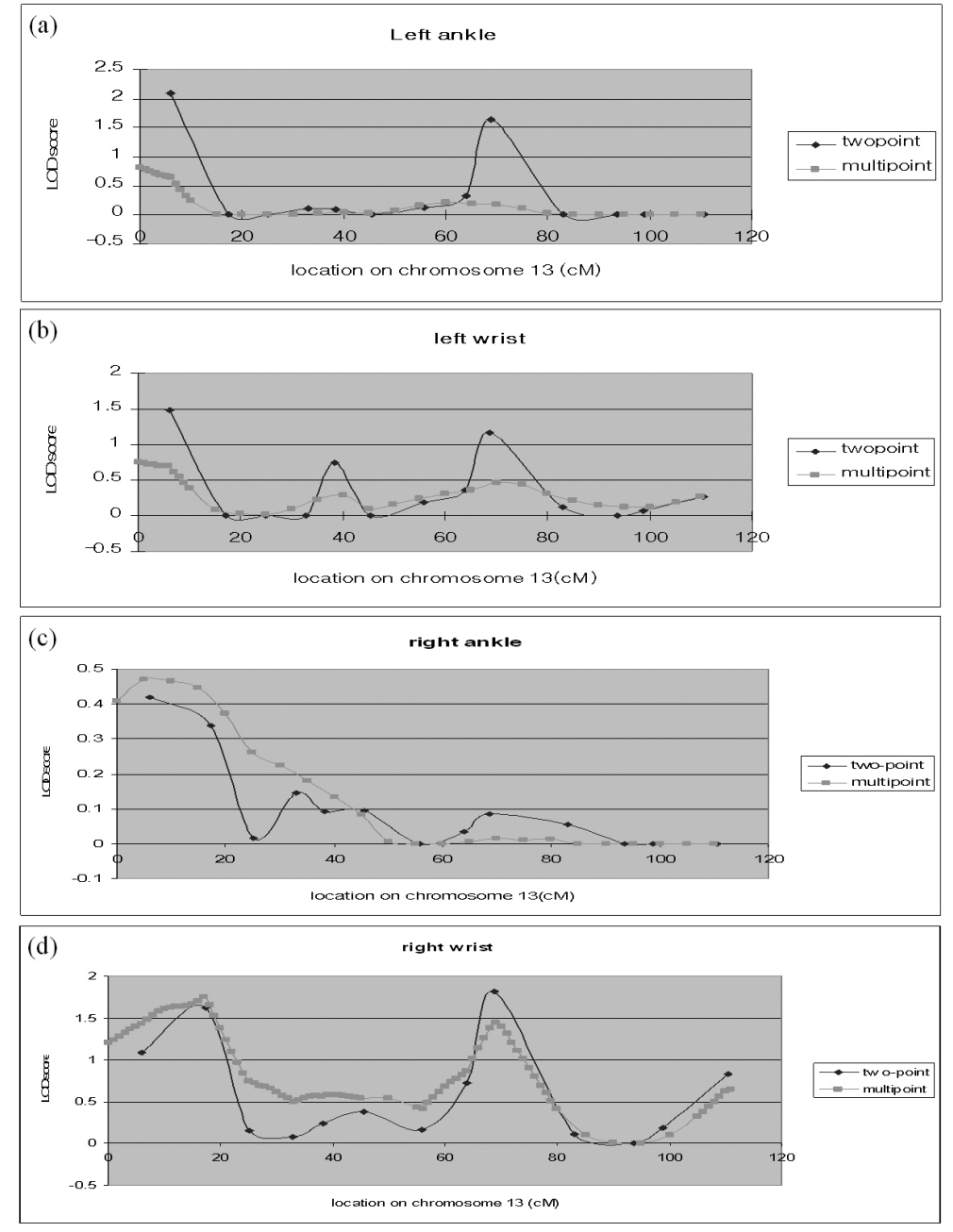

Fig. 2. LOD Score Plots. Variance component linkage analysis results show LOD scores of QTL for BMD at left ankle (a), left wrist (b), right ankle (c) and right wrist (d). Each figure shows the results derived two-point (circle) and multi-point (square) linkage analysis. 
Table 3. Maximun LOD scores from multipoint linkage analysis

\begin{tabular}{ccccl}
\hline $\begin{array}{c}\text { Chromosom } \\
\text { al location }\end{array}$ & Distance & $\begin{array}{c}\text { BMD } \\
\text { site }\end{array}$ & $\begin{array}{c}\text { Peak } \\
\text { LOD }\end{array}$ & $\begin{array}{c}\text { Peak or flanking } \\
\text { markers }\end{array}$ \\
\hline 13p13 & 0 & LA & 0.8229 & D13S175 \\
$13 p 13$ & 0 & LW & 0.7611 & D13S175 \\
$13 p 13$ & 0 & RA & 0.4103 & D13S175 \\
$13 p 11$ & 17 & RW & 1.7549 & D13S175, D13S217 \\
13q21 & 69 & RW & 1.4462 & D13S265, D13S779 \\
13q21 & 70 & LW & 0.4610 & D13S265, D13S779 \\
13q21 & 60 & LA & 0.2116 & D13S179, D13S156 \\
\hline
\end{tabular}

$B M D$, bone mineral density; LW, left wrist; LA, left ankle; $\mathrm{RW}$, right writst; $\mathrm{RA}$, right ankle.

one family (pedigree 1) had the highest LOD of 1.93 for D13S175 and 1.27 for D13S265. Another family (pedigree 39) had modest LOD scores of 0.27 for right ankle and 0.49 for right wrist.

Multipoint linkage analysis was performed with family 47 deleted due to technical problems. With the modified data it showed several suggestive QTLs for BMD. As shown in Table 3, the highest LOD score for left ankle was 0.8229 on $13 p 13$ at $0 \mathrm{cM}$ and the second highest score was 0.2116 on 13q21 on $60 \mathrm{cM}$ between markers D13S179 and D13S156. For BMD at left wrist, there were also two suggestive QTLs which were 0.7611 on $13 p 13$ and 0.4610 on 13q21 on $70 \mathrm{cM}$ between markers D13S265 and D13S779. QTLs for BMD of right ankle showed highest LOD score of 0.4103 on $13 \mathrm{p} 13$ at $0 \mathrm{cM}$. As for the right wrist $B M D$, we identified two significant QTLs; the highest LOD score being 1.7549 on 13p11at $17 \mathrm{CM}$ and the other having a LOD score of 1.4468 on $13 \mathrm{q} 21$ at $69 \mathrm{CM}$ between marker D13S170 and D13S265. The multipoint linkage results are shown in Fig. 2 and summarized in Table 3 along with the information content at each position

\section{Discussion}

We conducted a detailed genetic study of bone health measures using measures for the left and right wrists and ankles in members of multigenerational families from Mongolia. We show that in this population, heritability estimates for left wrist and left ankle exceeded 0.5 , and those for right wrist and ankle were somewhat lower. Linkage analysis localized potential QTL to two markers, D13S175 (6.03 cM) and D13S265 (68.73 cM).

This study provides a unique opportunity to parse genetic environmental factors that influence bone health measures using a large set of family data in Asian population. This population appears to have higher bone health measures compared with other Asian populations, highlighting the importance of environmental factors. Mongolian population appears to have higher BMD than other Asian populations, including Japanese and Chinese populations (Iki et al., 2001; Wu et al., 2004). Previous studies have consistently shown that Asian populations have lower BMD as compared with the population of European origin (Wand et al., 1997). Some of the findings confirm earlier studies. Comparison study of BMD by sex have shown that BMD is lower in females than in males by about $8 \sim 10 \%$. This difference between genders is the effect of men's larger bone size and greater bone strength. According to Looker and colleagues, men had significantly higher BMD values than women to around $10 \sim 15 \%$. However, when variables like bone size and bone strength were corrected, the difference was decreased to $1 \sim 8 \%$ (Looker et al., 2000).

The prevalence of osteoporosis in Mongolian women over age 50 was $19.2 \%$ and $21.6 \%$ in left and right wrist. This prevalence was lower than that reported in the Japanese Population Osteoporosis Study (Iki et al., 2001) of females over 50 with a $36.3 \%$ prevalence in the distal radius. On the other hand, the prevalence in our study was higher than that of other ethnicities with $10.0 \%, 5.0 \%, 13.3 \%, 15.6 \%$ and $15.1 \%$ in European, African, Asian, Hispanic, and native American origins, respectively, over 50 years measured in the distal forearm using DEXA (Barrett-Connor et al., 2005).

Heritability estimates exceed $50 \%$ after controlling for covariates that are important for bone health. Total additive genetic heritability $\left(\mathrm{H}^{2}\right)$ of the BMD were found to be more highly heritable in the left ankle and wrist than right. This is congruent with the expectation in that right wrist and ankle are likely to be affected by daily activities of living; therefore, the heritability, an estimate of genetic influence, will be underestimated.

Prior to multipoint analysis, we performed two-point analysis using measures of wrists and ankles. We found two significantly linked regions for BMD-LA and BMD-LW on marker D13S175 at $6.03 \mathrm{cM}$ and D13S265 at $68.73 \mathrm{cM}$ with a LOD greater than 1. In BMD-RA, the two markers showing the highest LOD were D13S175 and D13S217 but this result seems less feasible for two reasons: the heritability is too low $\left(\mathrm{H}_{\mathrm{r}}{ }_{\mathrm{r}}=0.03\right)$ and the highest LOD is less than 1. As for BMD-RW, the two highest LOD appeared in marker D13S265 and D13S217 (Fig. 2). This possibly suggests that genes may regulate BMD differently at different skeletal sites and that gene-environmental interactions may also play an important role. Nevertheless, all markers for high LOD of the four different traits were located between $6.03 \mathrm{cM}$ and $68.73 \mathrm{cM}$ on chromosome 13 . This suggests that 
there are some chromosomal regions highly linked to BMD between these two locations. Pedigree specific LOD revealed that pedigree 1 had the highest contribution to the overall LOD for D13S175 in BMD-LA and BMD-LW. Other families, pedigree 18, 21 and 139 also had high contributions. The mean BMD values for LA and LW in these families were slightly higher than the remaining families but the difference was not significant in paired t-test. The assumed reasons are that the number of these selected families was not big enough and that the BMD values were not corrected by variables such as sex and age. Therefore, a more sophisticated study design in subfamily analyses is needed to help understand the extent of genetic effects on BMD.

In addition to two-point linkage analysis, multipoint linkage analysis was performed. The strongest linkage signal was on $13 p 11$ ( $L O D=1.7549)$ for right wrist. This region has not been previously reported to have associations with BMD; nevertheless there is a candidate gene at $13 p 11$ for interleukin 17D. Interleukin 17 is known to affect osteoclastic resorption and mediate bone destruction, suggesting that it may be associated with BMD (Van Bezooijen et al., 1999).

The second strongest linkage in our study was on $13 \mathrm{q} 21.33$ at $69 \mathrm{cM}$ for right wrist $(\mathrm{LOD}=1.4462)$ which was also shown in left wrist with the LOD score of 0.4610 (Fig. 2). Chromosome 13q21 has been reported in other previous studies. Kammerer et al. (Kammerer et al., 2003) found a suggestive linkage on 13q14-13q22 in male Mexican Americans (LOD=3.46 of the trochanter and $L O D=2.51$ of the hip neck) which was close to our QTL region. There was another report of strong linkage (Hsu et al., 2007), which showed highest LOD score of 3.62 for BMD of lumbar spine on chromosome 13q21. However, this was performed as a sex-specific linkage analysis which only considered women. Although no obvious candidate genes are present in this region, there is another strong evidence for a QTL $(L O D=16.3)$ that affects femur BMD in femal mice (Beamer et al., 2001). This linkage was shown in a region homologous to human chromosome 13q14-21, which strongly suggests that these may be a relevant QTL. Deng et al. (Deng et al., 2002) also reported suggestive evidence ( $L O D=2.43$ ) for a QTL for spine BMD on 13q33-13q34, but this may represent a different QTL.

There were another suggestive QTLs for left ankle and wrist which showed linkages with $L O D=0.8229$ and 0.7611 each on $13 p 13$ at $0 \mathrm{cM}$. This corresponds with the results of the two-point analysis though the score was less significant in the multipoint linkage. Also, highest LOD score of right ankle was shown at the same region $13 \mathrm{p} 13$ at $0 \mathrm{cM}(\mathrm{LOD}=0.4103)$. To our knowledge, no previous linkage has been reported for BMD pheno- types in this region.

In summary, we identified three suggestive QTLs at chromosome 13p11, 13q21.33 and 13p13 in the Mongolian population. $13 q 21.33$ has been reported in previous studies, suggesting that there may be a relavant QTL in this region. The different findings between our study in Mongolian population and previous studies imply that genetic variation underlying BMD may depend on ethnicity or geographic location, though we should always consider that the differences in study design exist. Further studies such as fine mapping through linkage disequilibrium or positional candidate gene analysis will be required to definitively identify genes and their functional consequences that contribute to BMD.

\section{Acknowledgements}

This work was supported by a grant (M1030503000008N503-00000) from the Korean Ministry of Education, Science and Technology. This study was performed as part of an elective course of Seoul National University College of Medicine, at Columbia University Medical Center in New York, USA.

\section{References}

Barrett-Connor, E., Siris, E.S., Wehren, L.E., Miller, P.D., Abbott, T.A., Berger, M.L., Santora, A.C., and Sherwood, L.M. (2005). Osteoporosis and fracture risk in women of different ethnic groups. J. Bone Miner. Res. 20, 185-194.

Barthe, N., Basse-Cathalinat, B., Meunier, P.J., Ribot, C., Marchandise, X., Sabatier, J.P., Braillon, P., Thevenot, J., and Sutter, B (1998). Measurement of bone mineral density in mother-daughter pairs for evaluating the family influence on bone mass acquisition: A GRIO survey. Osteoporos, Int. 8, 379-384.

Beamer, W.G., Shultz, K.L., Donahue, L.R., Churchill, G.A., Sen, S., Wergedal, J.R., Baylink. D,J., and Rosen. C,J. (2001). Quantitative trait loci for femoral and lumbar vertebral bone mineral density in C57BL/6J and $\mathrm{C} 3 \mathrm{H} / \mathrm{HeJ}$ inbred strains of mice. J. Bone Miner. Res. 16, 11951206.

Deng, H.W., Xu, F.H., Huang, Q.Y., Shen, H., Deng, H., Conway, T., Liu, Y.J., Liu, Y.Z., Li, J.L., Zhang, H.T., Davies, K.M., and Recker, R.R. (2002). A whole-genome linkage scan suggests several genomic regions potentially containing quantitative trait loci for osteoporosis. J. Clin. Endocrinol. Metab. 87, 5151-5159.

Devoto, M., Specchia, C., Li, H.H., Caminis, J., Tenenhouse, A., Rodriguez, H., and Spotila, L.D. (2001). Variance component linkage analysis indicates a QTL for femoral neck bone mineral density on chromosome $1 \mathrm{p} 36$. Hum. Mol. Genet. 10, 2447-2452.

Hsu, Y.H., Xu, X., Terwedow, H.A., Niu, T., Hong, X., Wu, D., Wang, L., Brain, J.D., Bouxsein, M.L., Cummings, S.R., Rosen, C.J., and Xu, X. (2007). large-scale ge- 
nome-wide linkage analysis for loci linked to BMD at different skeletal sites in extreme selected sibships. J. Bone Miner. Res. 22, 184-194.

Iki, M., Kagamimori, S., Kagawa, Y., Matsuzaki, T., Yoneshima, $H_{\text {., }}$ and Marumo, F. (2001). Bone mineral density of the spine, hip and distal forearm in representative samples of the Japanese female population: Japanese Population-Based Osteoporosis (JPOS) Study. Osteoporos Int. 12, 529-537.

Jin, H., and Ralston, S.H. (2005). Genetics of osteoporosis. Curr. Rheumatol. Rep. 7, 66-70.

Jouanny, P., Guillemin, F., Kuntz, C., Jeandel, C., and Pourel, J. (1995). Environmental and genetic factors afftecting bone mass: Similarity of bone density among members of healthy families. Arthritis Rheum. 38, 61-67.

Kammerer, C.M., Schneider, J.L., Cole, S.A., Hixson, J.E., Samollow, P.B., O'Connell, J.R., Perez, R., Dyer, T.D., Almasy, L., Blangero, J., Bauer, R.L. and Mitchell, B.D. (2003). Quantitative trait loci on chromosomes $2 p, 4 p$, and $13 q$ influence bone mineral density of the forearm and hip in Mexican Americans. J. Bone Miner. Res. 18, 2245-2252.

Looker, A.C., Beck, T.J., and Orwoll, E.S. (2001). Does body size account for gender differences in femur bone density and geometry? J. Bone Miner. Res. 16, 12911299.

O'Connell, J.R., and Weeks, D.E. (1998). PedCheck: a program for identification of genotype incompatibilities in linkage analysis. Am J. Hum. Genet. 63, 259-266

Pocock, N.A., Eisman, J.A., Hopper, J.L., Yeates, M.G., Sambrook, P.N., and Eberl, S. (1987). Genetic determi- nants of bone mass in adults: A twin study. J. Clin. Invest. 80(3): 706-710.

Self, S.G., and Liang , K.Y. (1987). Asymptotic properties of maximum likelihood estimators and likelihood ratio tests under the nonstandard conditions. J. Am. Stat. Assoc. 82, 605-610.

Streeten, E.A., McBride, D.J., Pollin, T.I., Ryan, K., Shapiro, J., Ott, S., Mitchell, B.D., Shuldiner, A.R., and O'Connell, J.R. (2006). Quantitative trait loci for BMD identified by autosome-wide linkage scan to chromosomes $7 q$ and $21 \mathrm{q}$ in men from the Amish Family Osteoporosis Study. J. Bone Miner. Res. 21, 1433-1442.

Van Bezooijen, R.L., Farih-Sips, H.C., Papapoulos, S.E. and Lowik, C.W. (1999). Interleukin-17: a new bone acting cytoking in vitro. J. Bone Miner. Res. 14, 1513-1521.

Wang, M.C., Aguirre, M., Bhudhikanok, G.S., Kendall, C.G., Kirsch, S., Marcus, R., and Bachrach, L.K. (1997). Bone mass and hip axis length in healthy Asian, Black, Hispanic, and White American youths. J. Bone Miner. Res. 12, 1922-1935.

WHO Scientific Group (2003). Prevention and management of osteoporosis. (WHO technical report series No.921) (World Health Organization Press)

Wu, X.P., Liao, E.Y., Zhang, H., Dai, R.C., Shan, P.F., Cao, $X . Z$., Liu, S.P., and Jiang, Y. (2004). Determination of age-specific bone mineral density and comparison of diagnosis and prevalence of primary osteoporosis in Chinese women based on both Chinese and World Health Organization criteria. J. Bone Miner. Metab. 22, 382-391. 